\title{
Flexible scraping of viscous fluids
}

\author{
Jacopo Seiwert, David Quéré and Christophe Clanet $†$ \\ LadHyX, UMR 7646 du CNRS, École Polytechnique, 91128 Palaiseau CEDEX, France \\ Physique et Mécanique des milieux hétérogènes, UMR 7636 du CNRS, ESPCI, 75005 Paris, France
}

(Received 9 February 2012; revised 1 September 2012; accepted 19 October 2012)

We study the thickness $h_{d}$ of the liquid film left on a wet surface after scraping it with an elastic wiper (length $L$, rigidity $B$ ) moved at a velocity $V$. The scraper is clamped vertically at a given distance above the substrate, and $h_{d}$ is maximal when the tip of the scraper is just tangent to the surface. We show experimentally and theoretically that this maximum thickness is $h_{\max } \simeq 0.33 L\left(\eta V L^{2} / B\right)^{3 / 4}$, where $\eta$ is the liquid viscosity. The deposition law is found to be sensitive to the shape of the wiper: the film thickness can also be tuned by using wipers with a permanent curvature, and varying this curvature.

Key words: interfacial flows (free surface), low-Reynolds-number flows, thin films

\section{Introduction}

Scraping a fluid from a solid surface is a common situation in everyday life and in industrial processes, which is performed using a large variety of tools, from rigid paint scrapers to rubber squeegees. It is important to realize that wipers always deposit a wetting liquid film (of thickness $h_{d}$ ) behind them, and as such, scraping and coating are two faces of the same phenomenon.

On the one hand, $h_{d}$ needs to be minimized in cleaning applications (e.g. car wipers or floor squeegees), in order to decrease the drying time and to impose strong shearing stresses to remove dust particles. On the other hand, some coating processes work by removing an excess of liquid with a flexible blade (Kistler \& Schweizer 1997), and require precise control of the deposited film. For example, so-called knife or blade coatings have become the standard method for paper coating. In these set-ups, the paper is often horizontal and pre-coated with a large amount of aqueous solution. The excess fluid is then scraped off when the surface is run at high speed $\left(1-20 \mathrm{~m} \mathrm{~s}^{-1}\right)$ under a metering steel blade (100-500 $\mu \mathrm{m}$ thick), leaving a film of thickness in the 1-10 $\mu \mathrm{m}$ range. The scraper is held against the surface at an angle between 10 and $60^{\circ}$, with a fixed force that controls the coating. This provides an important advantage of the method: it allows a prescribed coating thickness to be maintained while increasing the speed of the process. As reported by Eklund (1984), due to the elasticity of the knife, the force-thickness relationship exhibits a complex behaviour: it qualitatively changes with the angle of the scraper, and can exhibit non-monotonic behaviours.

One of the first studies of these systems was that of Taylor (1962), who calculated analytically the viscous flow in a corner of arbitrary angle $\theta$, representing the flow 
under a rigid spatula moving at a constant velocity $V$ parallel to a planar substrate. A key feature of this calculation is the divergence of hydrodynamic stresses where the scraper and the surface meet, which explains why a small quantity of liquid is always left on the surface. If a rigid scraper is used to dry a surface, it must be held almost vertically with a lot of force in order to minimize $h_{d}$. However, such a device is impractical, since it does not conform to the surface to be cleaned. Real wipers are most commonly flexible, and bent against the substrate: elastic forces cannot be neglected, hydrodynamic forces deform the scraper and Taylor's model is not applicable.

Given the practical importance of blade coating, most previous studies focused on the interplay between elastic and hydrodynamic forces. In the simplest approach, the flow under the blade is calculated in the lubrication approximation and coupled to the elasticity of the scraper, and the resulting equations are solved numerically. Other phenomena have also been included in the calculations: capillary effects at the tip of the scraper, deformability of the substrate, non-Newtonian behaviour of the coating solution, erosion of the blades, etc. (Sullivan \& Middleman 1986; Pranckh \& Scriven 1990; Corvolan \& Saita 1995). More recently, Iliopoulos \& Scriven (2005) went one step further and removed the lubrication assumption. Despite the number of phenomena at play in 'real' processes, these models compare favourably with available data and recover in particular the non-monotonic behaviour reported by Eklund (1984).

Following another path, Saita (1989) proposed a simplified model, where the viscous stresses deforming the scraper are calculated as if the latter were straight. Considering two limit cases, he proposed an implicit expression for lower and upper limits of the coating thickness. Due to this assumption, these limits are only valid in the regimes where the scraper is almost straight.

Our goal is to gain some deeper understanding of these phenomena by taking a slightly different approach and simplifying the scraping or coating process. The experimental set-up is presented in $\S 2$, followed by experimental results in $\S 3$ and the model in $\S 4$. Possibilities for tuning the deposition law are finally discussed in $\S 5$.

\section{Experimental set-up and protocol}

Our set-up is sketched in figure 1(a). Before each experiment, the glass surface (s) to be coated (generally $30 \mathrm{~cm}$ long and $3 \mathrm{~cm}$ wide) is cleaned, dried, weighed, and pre-coated with a large excess of silicone oil (o) (a few millimetres thick). Silicone oils totally wet the surfaces and their viscosity $\eta$ can be varied over a large interval (here between 0.1 and $17.4 \mathrm{~Pa} \mathrm{~s}$ ), for fixed surface tension $\gamma=0.02 \mathrm{~N} \mathrm{~m}^{-1}$ and density $\rho=960 \mathrm{~kg} \mathrm{~m}^{-3}$. The solid is then drawn at a constant speed $V(0.7$ to $19.1 \mathrm{~mm} \mathrm{~s}^{-1}$ ) by a motor-driven stage, under the elastic scraper (w) of length $L$ (between 2 and $8 \mathrm{~cm}$ ) clamped vertically at an adjustable height $y_{0}\left(0<y_{0}<L\right)$ above the glass. The wipers of length $L$, width $b$ and thickness $t$ are cut from plastic sheets with $t=260 \mu \mathrm{m}$ or $t=380 \mu \mathrm{m}$. We determined their rigidities $B$ by measuring their deflection under their own weight when clamped horizontally (as described thoroughly in Mahadevan \& Keller 1999). The values found, 4.2 and $14.3 \mathrm{mN} \mathrm{m}$ respectively, are consistent (within $10 \%$ ) with the theoretical expression $B=E t^{3} / 12\left(1-v^{2}\right)$, with a Poisson ratio of $v=0.38$ and Young's modulus of $E=2.6 \times 10^{9} \mathrm{~Pa}$, typical for plastic materials such as Mylar. Concerning the width $b$, these elastic sheets are a few millimetres wider than the coated glass surface to ensure rejection of the excess liquid.

The scraper expels most of the oil initially present, leaving a liquid film of thickness $h_{d}$ ranging from $10 \mu \mathrm{m}$ to $1 \mathrm{~mm}$. As shown in figure 2 , the deposited quantity of 
(a)

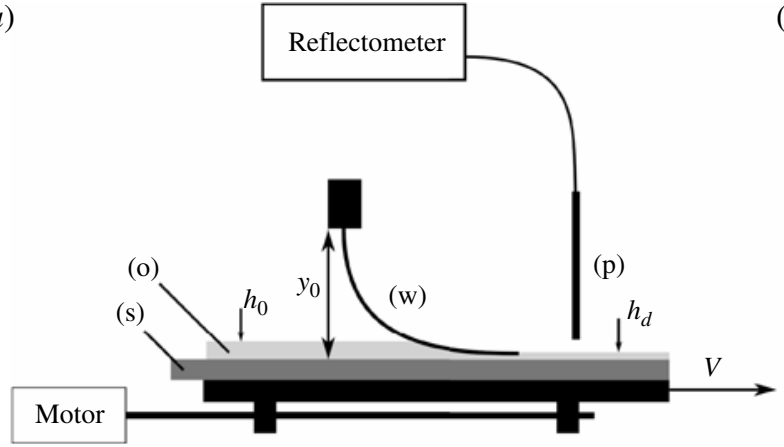

(b)

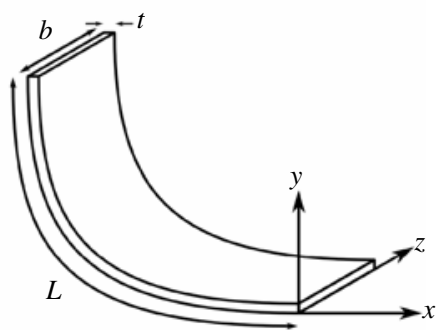

FIGURE 1. (a) Experimental set-up: a horizontal glass surface (s) coated with an excess of silicone oil (o) is run at a constant speed $V$ under a flexible wiper (w). The scraper, which is clamped vertically at a distance $y_{0}$ from the glass, thins the oil film to a thickness $h_{d}$, which is measured by reflectometry via a probe (p) and/or by weighing the coated substrate. (b) Characteristics of the elastic sheet.

liquid does not depend on the initial thickness $h_{0}$, provided that $h_{0}$ is several times thicker than the final coat. Depending on its expected value, $h_{d}$ is measured by reflectometry and/or by weighing. As for reflectometry, a beam of white light is shone via an optical fibre on the coated substrate, and the reflected light is collected. Its spectrum presents oscillations (due to the interference between the light reflected on the surface of the film, and the light reflecting off the substrate), which are fitted to determine the film thickness. This method allows a local $(0.5 \mathrm{~mm}$ lateral resolution) and precise (within 5\%) measure of thicknesses, and it works between 1 and $70 \mu \mathrm{m}$. Using this method, we scanned a few films to check the uniformity of the coating (figure $3 a$ ), whose thickness was found to be constant within a few per cent. When using this technique, we sampled the film thickness in typically five locations distributed on the film surface: median values are plotted on the graphs, with error bars spanning the entire recorded range. For thicker films (up to $1 \mathrm{~mm}$ ), we first removed oil spills stuck on the underside of the glass and the first few centimetres of the film. We then weighed the surface to determine the mass of deposited oil, and hence its average thickness. Figure $3(b)$ shows that the two techniques agree in the 30-70 $\mu \mathrm{m}$ range, but weighing produces inconsistent measures for thinner films (probably due to incomplete removal of oil from the glass), restricting this technique to thicker films.

\section{Experimental results}

One novelty of this kind of coating arises from the possibility of varying the pressure on the film, by manipulating the scraper's rigidity and height. We show in figure 4 how $h_{d}$ depends on the scraper's clamping height $y_{0}$. In our set-up, $y_{0}$ is a complex parameter since it controls both the loading of the blade and its shape: as sketched in the figure, the scraper goes from almost straight and vertical when $y_{0} \sim L$ to highly bent when $y_{0} \ll L$. Starting from a constrained wiper, $h_{d}$ first rises with $y_{0}$ as expected, since the force exerted by the scraper then decreases. It reaches a maximum of $h_{\max }(0.5 \mathrm{~mm}$ for these particular settings), which corresponds to a scraper whose tip is just tangent to the surface. Increasing $y_{0}$ even further results in a sharp decrease of the coating thickness. This somewhat counter-intuitive result (the scraper is less 


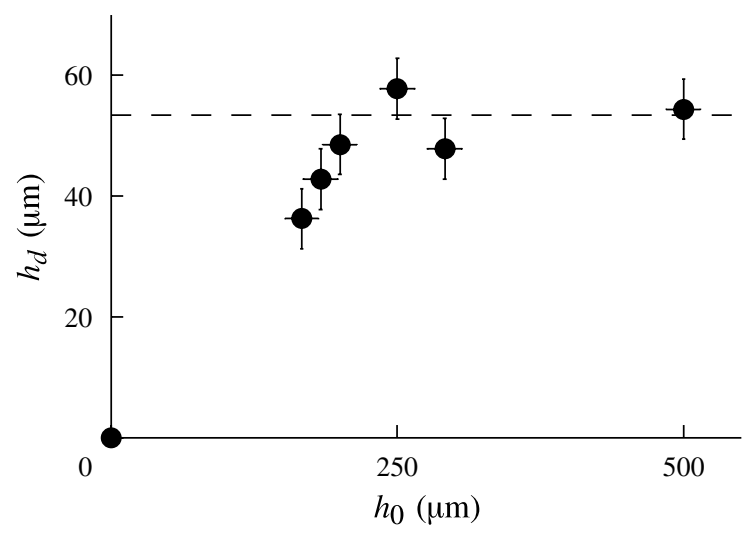

FigurE 2. For a given set of parameters $\left(B=4.2 \mathrm{mN} \mathrm{m}, L=4 \mathrm{~cm}, y_{0}=18.4 \mathrm{~mm}\right.$, $\left.\eta=0.98 \mathrm{~Pa} \mathrm{~s}, V=8.2 \mathrm{~mm} \mathrm{~s}^{-1}\right)$, the film thickness $h_{d}$ after scraping is measured as a function of the oil thickness $h_{0}$ initially on the surface. First $h_{d}$ increases with $h_{0}$, showing that the coating thickness can be limited by the quantity of liquid initially available. Once $h_{0}$ is approximately 5 times larger than the final coating, the curve reaches a plateau and $h_{d}$ no longer depends on the initial quantity of oil. All the measurements presented thereafter are performed in this plateau regime.
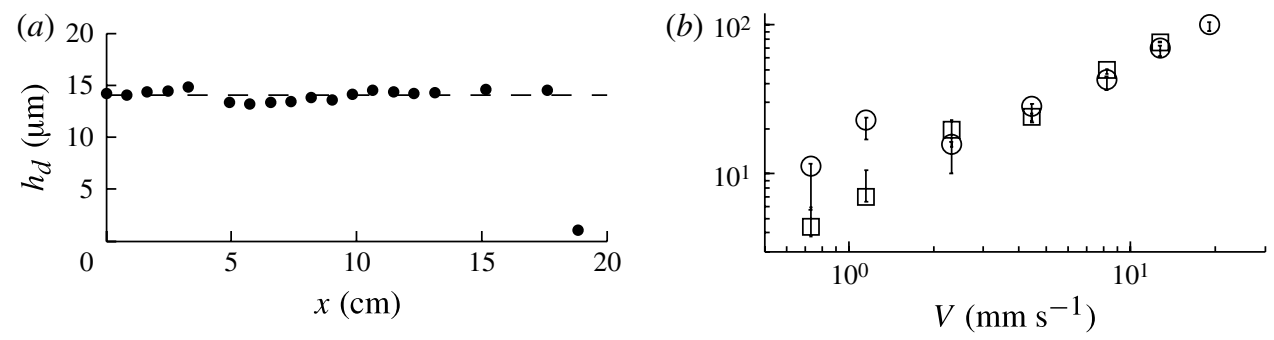

FIgURE 3. (a) Thickness $h_{d}$ at the centre line of a coated surface along its length $x$. In this graph, the scraper moved from right to left. This longitudinal profile was obtained by moving the substrate under the interferometer, and taking measurements at regular intervals (1 s). For this particular experiment, $B=7.4 \mathrm{mN} \mathrm{m}, L=8 \mathrm{~cm}, y_{0}=3.7 \mathrm{~cm}, \eta=0.98 \mathrm{~Pa} \mathrm{~s}$, $V=8 \mathrm{~mm} \mathrm{~s}^{-1}$. The liquid film is uniform within measurement accuracy (the round mean square of the profile is $4 \%)$. (b) $h_{d}$ for a given set of parameters $(B=4.2 \mathrm{mN} \mathrm{m}, L=4 \mathrm{~cm}$, $\left.y_{0}=18.4 \mathrm{~mm}, \eta=0.98 \mathrm{~Pa} \mathrm{~s}\right)$ as a function of the scraping velocity $V$, measured by reflectometry $(\square)$ or weighing $(\bigcirc)$. The two methods agree in the 30-70 $\mu \mathrm{m}$ range, whereas weighing deviates and becomes unreliable for thinner films.

and less constrained, so it exerts a smaller force on the liquid) arises from the very different shape of the scraper in this regime, the wiper meeting the surface at a finite angle that increases with $y_{0}$. Although our experiment cannot be directly compared to industrial blade coating apparatus, Eklund (1984) observed a similar non-monotonic dependence of the coating thickness on blade loading, in some operating regimes. From a practical standpoint, if the goal is to sweep liquid from a surface, we find that it is much easier to use an almost vertical scraper and apply little force, which is what decorators plastering a wall do intuitively when trying to remove an excess of plaster. Conversely, the deposition of an even (thick) coat of filler to cover a crack is best performed with the scraper highly bent and tangent to the wall. 


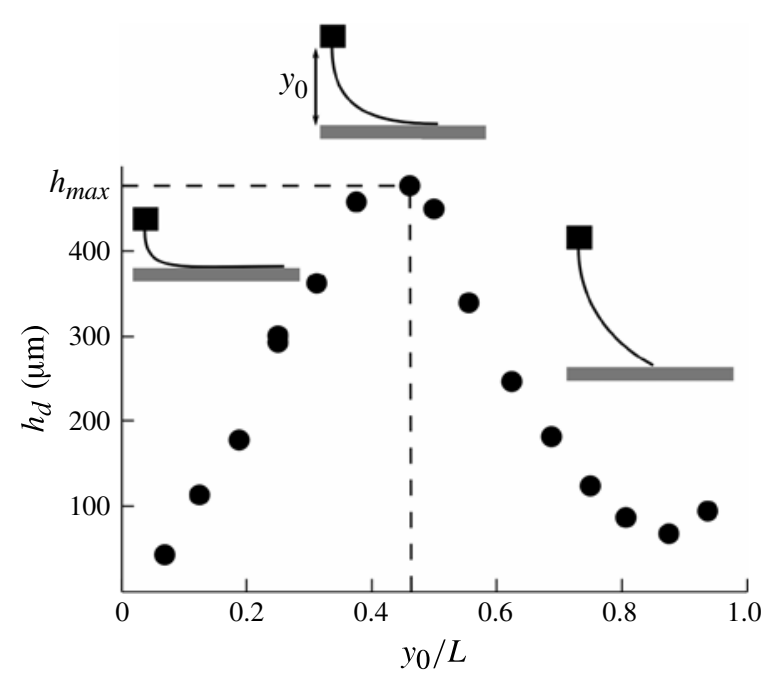

FIGURE 4. Deposited thickness $h_{d}$ as a function of the reduced clamping height $y_{0} / L$, for $V=8.2 \mathrm{~mm} \mathrm{~s}^{-1}, \eta=17.4 \mathrm{~Pa} \mathrm{~s}, B=4.2 \mathrm{mN} \mathrm{m}$ and $L=4 \mathrm{~cm}$. Error bars lie within the symbols. As sketched, small values of $y_{0}$ correspond to highly bent scrapers and thin films. As $y_{0}$ increases, the scraper straightens out and the film thickens by approximately one order of magnitude, before thinning by the same amount. The maximum thickness $h_{\max }$ is obtained for a wiper just tangent to the surface (middle sketch). In the decreasing region of the graph the wiper meets the surface with a non-zero angle. The rest of the study focuses on the maximum thickness $h_{\max }$, fixing $y_{0}=0.46 L$ (dashed lines).

The coating law in figure 4 qualitatively holds over the whole range of parameters that we explored. The coating thickness, however, obviously depends on $L, B, \eta$ and $V$. In order to characterize these variations, we focus in the rest of this study on the value of the maximum film thickness $h_{\max }$, and adjust for each experiment the scraper's height to make its tip tangent to the surface: classical thin beam elasticity shows that this imposes $y_{0} \simeq 0.46 L$ (Landau, Lifshitz \& Kosevich 1990), in agreement with the location of the maximum in figure 4. Although the clamping height of the scraper is set prior to the experiments, its shape is very slightly affected by the deposition of the liquid film, since $h_{\max } \ll y_{0}$.

Figure 5 summarizes our experimental data. Each curve corresponds to a particular scraper (i.e. given values of $L$ and $B$ ), and the deposited thickness $h_{\max }$ was measured when spanning the range of coating velocities $V$, and for three different viscosities: $\eta=0.1 \mathrm{~Pa} \mathrm{~s}$ (squares), $\eta=0.98 \mathrm{~Pa} \mathrm{~s}$ (circles), $\eta=17.4 \mathrm{~Pa} \mathrm{~s}$ (triangles). The different sets line up when plotted as a function of $\eta V$, emphasizing that viscous forces are responsible for entrainment. The three curves follow a power law of exponents $0.83 \pm 0.07,0.88 \pm 0.04$ and $0.75 \pm 0.06$ (bottom to top). The lowest curve (black symbols $)$ is for a relatively stiff and short scraper $(B=14.3 \mathrm{mN} \mathrm{m}, L=4 \mathrm{~cm}$ and accordingly $y_{0}=1.84 \mathrm{~cm}$ ). Using a more flexible scraper of the same length (grey symbols, $B=4.2 \mathrm{mN} \mathrm{m}$ ) results in a thicker coating. Using a slightly longer scraper (empty symbols, $L=6 \mathrm{~cm}$ ) also increases $h_{\max }$. Both these behaviours can be explained qualitatively if considering that elastic forces oppose entrainment. Indeed, decreasing $B$ lowers the elastic stresses, thus increasing $h_{\max }$. It is likely that when the length $L$ is increased the curvature is reduced (the scraper is less constrained since $y_{0}$ increases with $L$ ), again lowering elastic forces. 


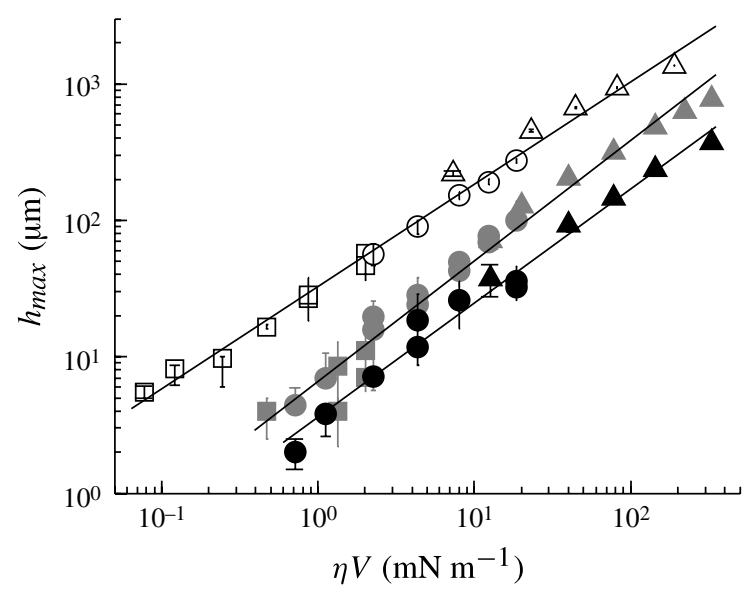

FIGURE 5. Maximum deposited thickness $h_{\max }$ for three different scrapers. Each curve presents data obtained by varying the coating velocity (between 0.7 and $19.1 \mathrm{~mm} \mathrm{~s}^{-1}$ ), for three different viscosities: $\eta=0.1 \mathrm{~Pa} \mathrm{~s}$ (squares), $\eta=0.98 \mathrm{~Pa} \mathrm{~s}$ (circles) and $\eta=17.4 \mathrm{~Pa} \mathrm{~s}$ (triangles). The three sets collapse if $h_{\max }$ is plotted as a function of $\eta V$. Black symbols correspond to a stiff and short scraper $(B=14.3 \mathrm{mN} \mathrm{m}, L=4 \mathrm{~cm})$. The deposited thickness increases for a more flexible one (grey symbols, $B=4.2 \mathrm{mN} \mathrm{m}$ and $L=4 \mathrm{~cm}$ ), or for a longer one (empty symbols, $B=4.2 \mathrm{mN} \mathrm{m}$ and $L=6 \mathrm{~cm}$ ). The experimental curves follow power laws (solid lines) with fitted exponents $0.83 \pm 0.07,0.88 \pm 0.04$ and $0.75 \pm 0.06$.

\section{Model}

\subsection{Scaling arguments for the value of $h_{\max }$}

When the tip of the scraper is tangent to the surface $\left(h_{d}=h_{\max }\right)$, our experiment has some similarities to dip-coating, one of the simplest coating processes. In dipcoating, the surface to be covered is withdrawn from a liquid bath at a constant velocity $V$. When a Newtonian fluid is used, Landau \& Levich (1942) and Derjaguin (1943) (LLD) showed that the thickness $h_{d}$ of the film scales like the capillary length $a=\sqrt{\gamma / \rho g}$ and increases with the capillary number $C a=\eta V / \gamma$, as $C a^{2 / 3}$. This behaviour results from a balance between viscous and capillary forces, the latter arising from the dynamic deformation of the meniscus. In this sense, the meniscus acts as a 'liquid wiper', so that deposition can be tuned by modifying its properties. This can be done chemically by adding surfactants (Ramdane \& Quere 1997; Shen et al. 2002), or geometrically by constraining it inside a tube (Bretherton 1961). In both these examples, the power law in capillary number is generally not affected by the meniscus modifications, which mainly impact the numerical factor in the law. Our experiment can be viewed as a variation on this celebrated study where the liquid meniscus has been replaced by an elastic one.

Throughout our range of parameters, the Reynolds number based on film thickness is much smaller than unity $\left(R e=\rho V h_{\max } / \eta=10^{-6}-10^{-2}\right)$, justifying the fact that viscous forces dominate inertia. In addition, the capillary number $C a=\eta V / \gamma$ is large $\left(10-10^{4}\right)$, suggesting that surface tension may be neglected. Film thickness thus results from a balance between viscous and elastic forces. In the absence of flow, the tip of the scraper is tangent to the surface to be coated. Since its curvature at the tip 


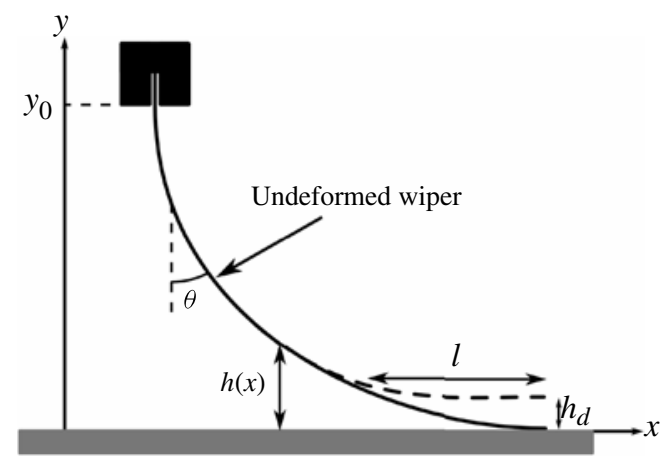

FIGURE 6. In the absence of flow, the shape of the wiper is determined by elasticity and geometrical constraints. In particular, as its end is free, it approaches the surface with zero curvature. When liquid is entrained, it lifts the scraper. Hydrodynamic forces are negligible if the height of the scraper is greater than $h_{\max }$, which determines the length $l$ of the deformed region.

must vanish to satisfy the elastic boundary condition, we deduce that its shape close to the contact goes as $y \sim-x^{3} / L^{2}$. We discuss the coating in the limit of 'small' velocities where the shape of the elastic sheet is weakly affected $\left(h_{\max } \ll L\right)$. In this limit, the fluid is forced in the gap under the wiper, which gets locally deformed by hydrodynamic forces, as sketched in figure 6. The gap at the tip is of order $h_{\max }$, and this allows us to estimate the horizontal extent $l$ of the region of deformation, i.e. $h_{\text {max }} \sim l^{3} / L^{2}$, or

$$
l \sim\left(h_{\max } L^{2}\right)^{1 / 3} .
$$

This deformation of the elastic sheet results from the balance between the elastic force $f_{e l}$ and the viscous force $f_{h} \sim \eta V / h_{\max }^{2}$. Since the curvature of the scraper is of order $1 / L$, it exerts a total force per unit length of order $B / L^{2}$, which generates an 'elastic' pressure gradient $f_{e l} \sim B / l^{2} L^{2}$. Balancing $f_{h}$ and $f_{e l}$ and using (4.1) yields a prediction for the deposited film thickness:

$$
h_{\max } \sim L^{5 / 2}\left(\frac{\eta V}{B}\right)^{3 / 4} \sim L C e^{3 / 4} .
$$

In this expression, the number $C e \sim \eta V L^{2} / B$ compares viscous and elastic forces. $C e$ plays the role of the capillary number in the LLD theory, replacing the surface tension by the elastic energy per unit area $B / L^{2}$. The exponent is also different $(3 / 4$ instead of $2 / 3$ ), which arises from the specific boundary condition related to the presence of a bent elastic solid. Equation (4.2) captures the different trends found in figure 5, and particularly the observed scaling law in $\eta V$. To address the other variations, in figure 7 we have plotted $h_{\max }$ as a function of the thickness predicted by (4.2), for the entire set of data. Thickness $h_{\max }$ is indeed proportional to $L C e^{3 / 4}$ with a numerical coefficient of $0.10 \pm 0.05$. A slight deviation is observed when reaching millimetric thicknesses. This might be an artifact due to the difficulty of dealing with such thick films on a narrow surface, or a sign that our assumption of small disturbance no longer holds. Indeed, the model assumes $h_{\max } \ll L$; from (4.2) this implies $C e^{3 / 4} \ll 1$, which becomes questionable for the highest $C e$ explored. 


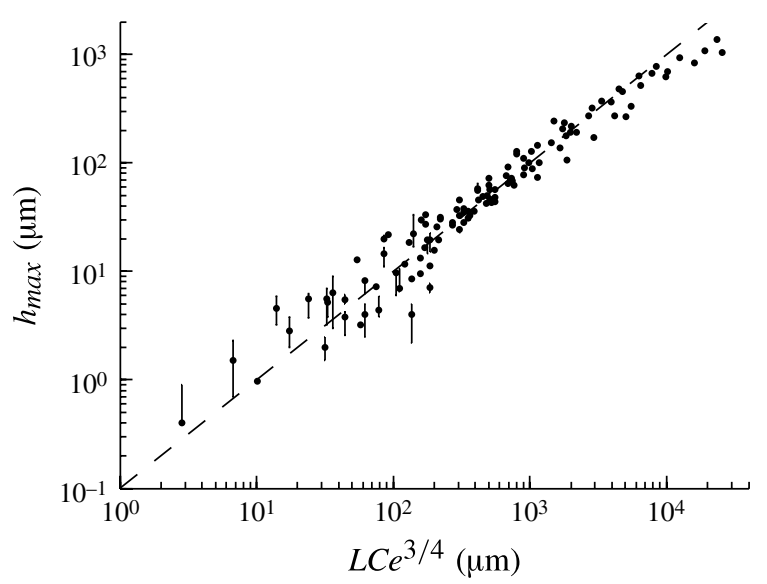

FIGURE 7. Comparison between the measured thickness $h_{\max }$ and (4.2). The line has a slope 1 and the fit allows us to determine the numerical coefficient $0.10 \pm 0.05$ (solid line). Data correspond to three viscosities $(\eta=0.1,0.98$ and $17.4 \mathrm{~Pa} \mathrm{~s})$, two bending rigidities $(B=4.2$ and $14.3 \mathrm{mN} \mathrm{m})$, several scraper lengths $(L=2,4,6$ and $8 \mathrm{~cm})$ and velocities $V$ between 0.7 and $19 \mathrm{~mm} \mathrm{~s}^{-1}$, so that four orders of magnitude could be explored.

\subsection{Theory for the maximum coating thickness $h_{\max }$}

We now consider the shape of the scraper in greater depth. We separate the undeformed region ('static scraper'), whose shape is known from classical elastic theory, from the deformed region ('dynamic scraper'), whose shape is given by a balance between elastic and hydrodynamic forces. We further assume that the dynamic scraper lies almost flat $\left(h_{x} \ll 1\right.$ in the notation of figure 6), and use the lubrication approximation. The fluid velocity is parallel to the coated surface, and it only varies in the vertical direction: $\boldsymbol{v}=v(y) \boldsymbol{e}_{x}$. As a result, the pressure is independent of $y$ and can be deduced from the continuity of stress at the scraper's surface. In the small slope limit, the pressure just under the blade is $p=p_{\text {atm }}+B h_{x x x x}$ (Hosoi \& Mahadevan 2004). The Stokes equation relates $v$ to the scraper profile $h(x): \eta v_{y y}=p_{x}=B h_{x x x x x}$. It is integrated with no-slip conditions at the moving coated surface $(v(0)=V)$ and on the wiper $(v(h)=0)$, yielding the liquid flux (per unit width). The flux is evaluated in the deposited film, where it is $q=V h_{\max }$. Hence we obtain

$$
q=-B h_{x x x x x} \frac{h^{3}}{12 \eta}+\frac{V h}{2}=V h_{\max } .
$$

Using $h_{\max }$ to scale the thickness $\left(Y=h / h_{\max }\right)$ and $l=h_{\max } /\left(6 \eta V h_{\max }^{2} / B\right)^{1 / 5}$ to scale $x(X=x / l)$, we get a non-dimensional equation for the profile of the dynamic scraper:

$$
Y^{(5)}=\frac{Y-2}{Y^{3}}
$$

Boundary conditions are needed to solve (4.4). The dynamic scraper is connected to the entrained film and thus becomes horizontal for $x \rightarrow \infty$, which gives $Y(x \rightarrow \infty)=2$. This value of 2 (instead of the 1 in the LLD problem) comes from the difference in boundary conditions under the scraper (no slip on the top boundary yielding a Poiseuille flow) and in the film (no tangential stress yielding a plug flow). To take this boundary condition into account in the numerical integration, we look for 

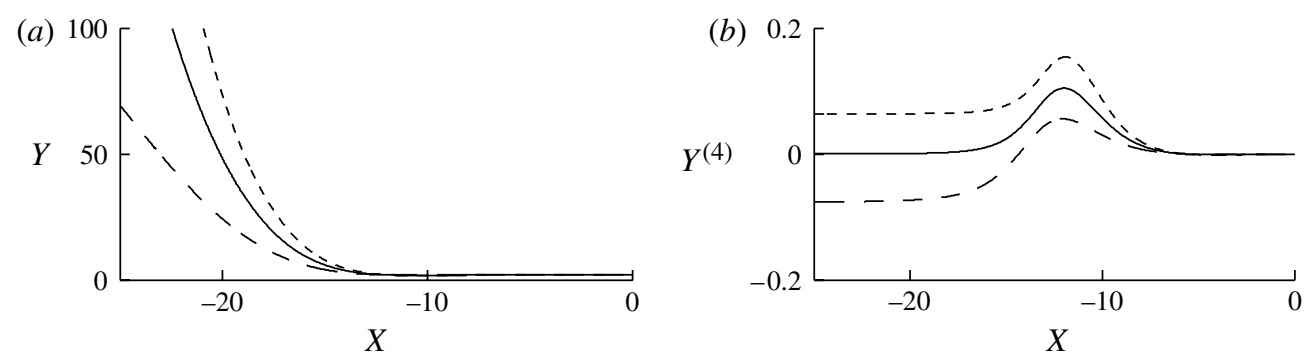

FIGURE 8. Profiles of the dynamic scraper, obtained from integration of (4.4) for different values of $\epsilon_{0}: 0.0012$ (solid lines), 0.0017 (dashed) and 0.0007 (dotted). $X$ and $Y$ are dimensionless coordinates defined in the text. $Y$ diverges for decreasing $X$, and $Y_{X X X X}$ converges towards a constant value controlled by $\epsilon_{0}$.

an asymptotic solution of (4.4) of the form $Y=2+\epsilon$, with $\epsilon \ll 1$. At first order, the general solution is given by $\epsilon(x)=a_{i} \mathrm{e}^{r_{i} X}$, where $r_{i}$ are the fifth roots of 1 . Requiring the solution to be real and to vanish for $X \rightarrow \infty$ cancels four of the five $a_{i}$ coefficients. Thus $\epsilon(X)$ depends only on one free parameter $\epsilon_{0}$, and may be written as

$$
\epsilon(X)=\epsilon_{0} \mathrm{e}^{-(\cos \pi / 5) /\left(8^{1 / 5}\right) X} \cos \left(-\frac{\sin \pi / 5}{8^{1 / 5}} X\right) .
$$

Figure 8 shows different profiles obtained from integration of (4.4) (with initial conditions computed from expression (4.5)), for different values of $\epsilon_{0}$. In the limit $X \rightarrow-\infty, Y$ diverges and $Y_{X X X X}$ tends towards a constant (which depends on $\epsilon_{0}$ ), as expected from (4.4). The value of this constant is selected from the matching with the static scraper, which is imposed to analyse its shape. It is given by the elastica equation (Landau et al. 1990), and can be written in curvilinear coordinates (see figure 6 for notation):

$$
\frac{\mathrm{d}^{2} \theta}{\mathrm{d} s^{2}}=-\frac{f_{e l}}{B} \sin \theta .
$$

Equation (4.6) is solved with the usual conditions: $\theta(s=0)=0$ (vertical clamping) and $\theta_{s}(s=L)=0$ (free end). Here $f_{e l}$ is the force exerted by the scraper's tip on the surface, and it is not known a priori: it is a free parameter chosen to satisfy the geometric constraint given by $y_{0}$. In the configuration of maximum coating thickness $\left(y_{0}=0.46 L\right)$, we have $f_{e l}=3.43 \mathrm{~B} / \mathrm{L}^{2}$ : we recover the scaling used in $\$ 4.1$. Equation (4.6) reduces close to the coated surface to $h_{x x x}=-f_{e l} / B$ and $h_{x x x x}=0$, providing a matching constraint with the dynamic scraper on the third derivative. Although it arises from mathematical considerations, this constraint has a clear physical meaning: in the small slope limit, $h_{x x x}\left(x_{0}\right)$ is proportional to the force applied on the scraper for $x>x_{0}$. The matching condition simply expresses that the hydrodynamic force under the deformed region balances the force exerted by the scraper. This condition is met on the 'left side' of the dynamic meniscus (that is, for $x \rightarrow-\infty$ ) for one particular value of $\epsilon_{0}$ (solid lines in figure 8), yielding $Y_{X X X}(x \rightarrow-\infty)=-0.478$, or in dimensional units $h_{x x x}(x \rightarrow-\infty)=-0.478 h_{\max } / l^{3}$. Equating this quantity with $-3.43 B / L^{3}$ gives the deposition law

$$
h_{\max } \simeq 0.33 L C e^{3 / 4}
$$




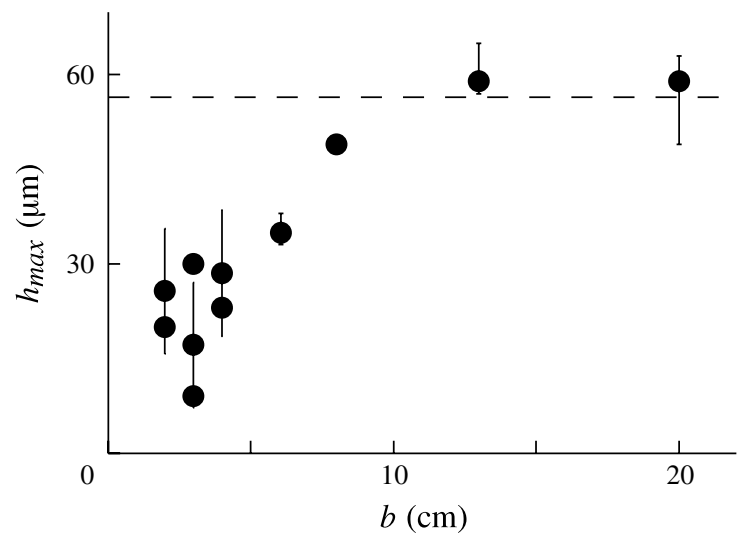

FIGURE 9. Deposited thickness $h_{\max }$ as a function of the scraper's width $b$, for a typical set of parameters $\left(\eta=0.98 \mathrm{~Pa} \mathrm{~s}, V=19 \mathrm{~mm} \mathrm{~s}^{-1}, B=4.2 \mathrm{mN} \mathrm{m}\right.$ and $\left.L=2 \mathrm{~cm}\right)$ and various scraper widths $b$. First $h_{d}$ increases with $b$, then reaches a value close to the prediction of the model (4.7) shown by the dashed line.

where $C e=\eta V L^{2} / B$ must be smaller than unity to satisfy the assumptions made in this calculation.

\subsection{Comparison with experimental data}

Equation (4.7) complies with the previous scaling arguments (4.2). It also provides a numerical coefficient for the law, which is found to be of the correct order of magnitude, yet significantly bigger than measured (0.33 instead of 0.10$)$. The discrepancy may come from different factors, but the dominant cause seems to be related to the assumption of invariance in the transverse direction $z$. This is certainly true when the width $b$ of the experiment tends to infinity. When $b$ is finite, though, liquid escaping from the side of the scraper may decrease the deposited thickness. This was tested by performing coating experiments with different scraper widths ranging from 2 to $20 \mathrm{~cm}$. Figure 9 shows that the deposited thickness first increases with $b$ until $b \sim 12 \mathrm{~cm}$. The coating experiments previously shown were all performed with $b=3 \mathrm{~cm}$, and thus lie in this varying regime of important finite-width effects. For $b>12 \mathrm{~cm}$, however, $h_{d}$ reaches an asymptote close to the value calculated by our theory ((4.7), dashed line in graph).

The finite width of the scraper should also affect the coating law. Indeed, letting $P$ denote the typical pressure under the scraper, the lateral flux of liquid scales as $P h_{\text {max }}^{3} l / \eta b$, whereas the longitudinal flux scales as $P h_{\max }^{3} b / l \eta$. For a given scraper, $l$ increases with the deposited thickness, and deviations from the two-dimensional case should increase as well. This effect is subtle and seems to lie within experimental error; the slight concavity of the deposition curve (figure 5) might reflect this idea.

\section{Tuning the deposition law}

Our model suggests that the deposited thickness depends on the shape of the scraper near its tip, because this determines the length $l$ of the deformed region over which the elastic force is distributed. In the present work, the 'elastic meniscus' has a vanishing curvature, but would we expect different coating laws if the scraper were curved? To answer this question, we ran a series of experiments using scrapers with a finite radius 

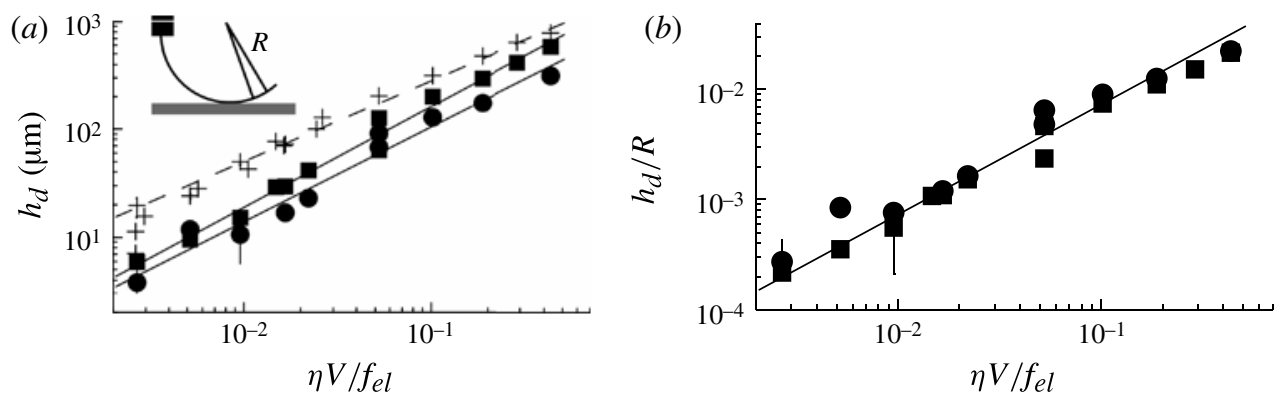

FIGURE 10. Deposited thickness $h_{d}$, with scrapers having a spontaneous radius of curvature $R=1.4 \mathrm{~cm}$ (circles) and $R=2.7 \mathrm{~cm}$ (squares). The elastic force $f_{e l}$ exerted by the scraper is the same for every experiment. (a) Solid lines represent power law fits, of exponents $0.88 \pm 0.15$ and $0.94 \pm 0.11$. The cross symbols and dashed lines are for a straight scraper and serve as a reference. $(b)$ Data for curved scrapers collapse when plotted in dimensionless form as suggested by (5.1). The solid line is a linear fit, with a coefficient 0.07 .

of curvature. These were cut from a flat sheet of $250 \mu \mathrm{m}$ thick Lexan, rolled inside a metallic pipe of centimetric diameter, heated to $140{ }^{\circ} \mathrm{C}$ for $3 \mathrm{~h}$, and then cooled. Once removed, the scrapers exhibit a permanent radius of curvature $R(1.4$ and $2.7 \mathrm{~cm}$ for the two wipers tested). For ease of comparison with previous experiments, the height of clamping was adjusted manually for each scraper to match the elastic force exerted by a given scraper in the tangent configuration discussed above (32 $\mathrm{g}$ equivalent for $L=4 \mathrm{~cm}, B=4.2 \mathrm{mN} \mathrm{m}$ and $b=3 \mathrm{~cm}$ ).

The resulting coating curves, displayed in figure 10 , are power laws in $\eta V$, with exponents of $0.88 \pm 0.15$ and $0.94 \pm 0.11$, differing from the previous scaling $(3 / 4$ in (4.7)). Moreover, the deposited thickness increases with the radius of curvature of the scraper. Scaling arguments similar to those leading to prediction (4.2) can explain why. The only difference is the shape of the scraper near its tip, now of constant curvature $1 / R$, which modifies the evaluation of $l$. We now have $h_{d} \sim l^{2} / R$, or $l \sim \sqrt{h_{d} R}$. This yields a new scaling for the deposited thickness:

$$
h_{d} \sim R \frac{\eta V}{f_{e l}} .
$$

Equation (5.1) is compatible with the $\eta V$ dependence observed in figure 10(a). The governing length scale of the problem is now the radius of curvature $R$ instead of the length of the scraper, which explains why $h_{d}$ increases with $R$. The data collapse when plotted in the dimensionless form suggested by (5.1) (see figure 10b), confirming the robustness of the scaling arguments developed here.

\section{Conclusions and perspectives}

We presented a set-up to study the scraping of viscous fluids by an elastic wiper, and to measure the thickness of the residual film. In the regime we explored, coating is controlled by a balance between viscous and elastic forces, so that it depends on the liquid viscosity, the deposition speed and the length, rigidity, shape and confinement of the scraper.

The wiper shape and the force it exerts on the liquid film are related and controlled by the clamping height. We found experimentally that the deposited thickness is maximized when the tip of the scraper is tangent to the solid, and we focused on 
understanding what parameters control this maximum value. Using scaling arguments, we explained why it varies like $L C e^{3 / 4}$ (where $C e=\eta V L^{2} / B$ compares viscous and elastic forces), and calculated the coefficient of this power law.

As a result of this analysis, the coating thickness is expected to depend on the shape of the scraper. It can thus be modified by using a blade with a given curvature. In this case, similar scaling arguments allowed us to predict a novel coating law, which was checked experimentally.

Future efforts will aim at understanding the dependence of the deposited thickness on the clamping height of the scraper (figure 4). The theory developed here for $h_{\max }$ will be adapted to explain the configuration where the scraper is closer to the surface. The opposite regime, where the scraper meets the surface at an angle, is more mysterious. As showed by Taylor (1962), the existence of this finite angle can have a dramatic effect on the coating thickness, which we shall now try to check experimentally.

Lastly, the present study leads us to consider a related and even more common coating situation, which seems to present new challenges: painting with a brush. One might think that the situation is similar to blade coating, and that the thickness of paint left on the surface is controlled by the flexibility of the bristles. A first difference is the importance of capillarity, which holds the paint in the brush. Moreover, this reservoir of paint has a limited volume, and the deposited thickness does vary with its depletion.

\section{REFERENCES}

Bretherton, F. P. 1961 The motion of long bubbles in tubes. J. Fluid Mech. 10, 166-188.

Corvolan, C. M. \& Saita, F. A. 1995 Blade coating on a compressible substrate. Chem. Engng Sci. 50, 1769-1783.

DeRJAGuin, B. 1943 On the thickness of the liquid film adhering to the walls of a vessel after emptying. Acta Physicochim. USSR 20, 349-352.

EKLUND, D. E. 1984 Influence of blade geometry and blade pressure on the appearance of a coated surface. In Proceedings of the 1984 TAPPI Coating Conference, pp. 37-43.

Hosoi, A. E. \& Mahadevan, L. 2004 Peeling, healing, and bursting in a lubricated elastic sheet. Phys. Rev. Lett. 93, 137802.

Iliopoulos, I. \& SCRIVEN, L. E. 2005 A blade coating study using a finite-element simulation. Phys. Fluids 17, 127101.

Kistler, S. F. \& Schweizer, P. M. 1997 Liquid Film Coating. Chapman \& Hall.

LAndau, L. \& Levich, B. 1942 Dragging of a liquid by a moving plate. Acta Physicochim. USSR 17, 42-54.

Landau, L., Lifshitz, E. \& Kosevich, A. 1990 Theory of Elasticity. Mir.

Mahadevan, L. \& Keller, J. B. 1999 Periodic folding of thin sheets. SIAM Rev. 41, 115-131.

PrANCKH, F. R. \& SCRIVEN, L. E. 1990 Elastohydrodynamics of blade coating. AIChE J. 36, 587-597.

RAmdane, O. O. \& Quere, D. 1997 Thickening factor in Marangoni coating. Langmuir 13, 2911-2916.

SaItA, F. A. 1989 Simplified models of flexible blade coating. Chem. Engng Sci. 44, 817-825.

Shen, A. Q., Gleason, B., McKinley, G. H. \& Stone, H. A. 2002 Fiber coating with surfactant solutions. Phys. Fluids 14, 4055-4068.

Sullivan, T. M. \& Middleman, S. 1986 Film thickness in blade coating of viscous and viscoelastic liquids. J. Non-Newtonian Fluid Mech. 21, 13-38.

TAYLOR, G. I. 1962 On scraping viscous fluid from a plane surface. In Miszellaneen der Angewandten Mechanik (Festschrift Walter Tollmien), pp. 313-315. 